\title{
What attitudes to risk underlie distortion risk measure choices?
}

Jaume Belles-Sampera • Montserrat Guillen • Miguel

Santolino

\begin{abstract}
Understanding the attitude to risk implicit within a risk measure sheds some light on the way in which decision makers perceive losses. In this paper, a two-stage strategy is developed to characterize the underlying risk attitude involved in a risk evaluation, when executed by the family of distortion risk measures. First, we show that aggregation indicators defined for Choquet integrals provide information about the implicit global risk attitude of the agent. Second, an analysis of the distortion function offers a local description of the agent's stance on risk in relation to the occurrence of accumulated losses. Here, the concepts of absolute risk attitude and local risk attitude arise naturally. An example is provided to illustrate the usefulness of this strategy for characterizing risk attitudes in an insurance company.
\end{abstract}

Keywords Risk management · Tolerance · Risk behavior · GlueVaR

JEL classification: C02, D81.

$\overline{\text { J.Belles-Sampera } \cdot \text { M.Guillen }} \cdot$ M.Santolino

Riskcenter-IREA, University of Barcelona, Av.Diagonal, 690 -08034- Barcelona, Spain 


\section{Introduction}

\subsection{Motivation}

Tools designed to provide adequate risk measurements are needed by both decision-making agents and regulatory agents, who require information about potential losses within a probabilistic framework. As such, the choice of a risk measure plays a central role in decision-making in many areas including health, safety, environmental, adversarial and catastrophic risks (Cox Jr., L.A., 2013, MacKenzie, 2014). Many different risk measures are available to practitioners, but the selection of the most suitable risk measure for use in a given context is generally controversial. A key element in characterizing a risk measure is the underlying risk attitude that is assumed when this measure is used for risk assessment. Clearly, therefore, in selecting the best measure, the practitioner is concerned with how a particular measure matches up with the alternatives. However, this simple question is only satisfied with a complex answer.

Consider the Value-at-Risk (VaR) and Tail Value-at-Risk (TVaR), probably the most common measures used in assessing risk. Suppose $\alpha$ is the confidence level, which reflects the degree of tolerance to undesirable events. The $\operatorname{VaR}_{\alpha}(X)$ is the $\alpha$-quantile of loss $X$, while the $\operatorname{TVaR}_{\alpha}(X)$ averages quantiles ranging from the $\alpha$-quantile to the maximum (the $100 \%$-quantile) of $X$. Based on these definitions, it seems obvious that these two quantile-based risk measures can be directly compared in terms of their respective conceptions of risk using their associated confidence levels. For instance, the Value-at-Risk measure provides for a concept of risk associated with a barrier, beyond which the decision maker assumes catastrophe lies (Alexander and Sarabia, 2012). A Value-at-Risk measure at a 95\% confidence level presents a lower resistance to undesirable events than a VaR measure at a $99 \%$ level. This also holds for $\mathrm{TVaR}_{\alpha}(X)$. Comparisons of $\mathrm{VaR}$ and $\mathrm{TVaR}$ measures can likewise be readily undertaken when their respective confidence levels are fixed and equal. Given an $\alpha$-confidence level, the $\operatorname{TVaR}_{\alpha}(X)$ is always greater or equal than the $\operatorname{VaR}_{\alpha}(X)$. However, a direct comparison cannot be made if the VaR and the TVaR risk measures have different confidence levels. For example, imagine a decision maker wishes to compare the implicit risk attitude of the $\operatorname{TVaR}_{95 \%}(X)$ and the $\operatorname{VaR}_{99 \%}(X)$. In this instance, it is not im- 
mediately obvious which of these two risk measures offers the greatest risk tolerance. Furthermore, if the decision maker wants to know the risk attitude of a measure other than that of these two quantile-based measures, comparisons are even less intuitive.

Here, we focus on the family of distortion risk measures, where the VaR and TVaR can be understood as two particular cases. A battery of instruments is developed to facilitate the comparison of the risk attitude of distortion risk measures from both global and local perspectives. The results afford new elements for determining the suitability of a particular distortion risk measure in comparison with other available options. They also allow an agent to determine which risk measure provides the most risk tolerant behavior.

\subsection{Attitude towards risk}

This article seeks to contribute to the study of attitudes towards risk in the assessment of risk. The study analyses the risk perception that is implicit when an agent applies a particular distortion risk measure. The characterization of the implicit attitude towards risk in a given distortion risk measure is carried out by means of the computation of aggregation indicators and an analysis of the distortion function. The combination of these two instruments provides a precise portrait of the underlying risk position of a decision-maker when selecting a particular risk measure for risk assessment.

Distortion risk measures can be represented mathematically as a class of Choquet integrals Wang, 1995. Belles-Sampera et al., 2013, Grigorova, 2014). One way in which to describe the characteristics of Choquet integrals is to use a set of aggregation indicators, which provide information about features of the underlaying aggregation operator (Marichal, 2004, Beliakov et al., 2007, Belles-Sampera et al., 2014c, Yager, 2000, Kojadinovic et al. 2005, Grabisch et al., 2009). Here, we investigate the quantitative information related to the overall risk attitude associated with the risk measure as provided by the aggregation indicators. It is our contention that these indicators are useful for characterizing the global perception of risk implicit in the risk measure choice. 
It is reasonable to suppose that decision-makers do not worry about all random event losses in the same way. Decision makers frequently treat different random events distinctly (note that some of these events can represent benefits or affordable losses). Therefore, the global vision of risk embedded in a risk measure has to be completed with local information. In this paper, we define a quotient function, based on the distortion function associated with the risk measure, in order to characterize the local vision of risk. The quotient function is graphically analyzed to investigate the risk attitude of the agent at any point in the survival distribution function when using a certain risk measure. The graphical evaluation of the risk-appetite pattern of a manager in the range of feasible values is the basis of the definition of two concepts: absolute risk attitude and local risk attitude.

The attitudes to risk implicit within three particular distortion risk measures are studied. Our attention is focused on the characterization of the attitudes toward risk of the VaR, TVaR and a class of four-parameter distortion risk measures that are called GlueVaR (Belles-Sampera et al., 2014a). The high flexibility of the GlueVaR distortion measures allows different specific attitudes to be reflected. We examine the additional risk information provided by these risk measures and their usefulness for decision makers.

An illustrative example of the risk attitude characterization implicit in a distortion risk measure is included in this article. The European insurance regulatory framework serves as an excellent example of the choice of a compulsory risk measure, i.e. VaR $99.5 \%$. However, insurers implement other choices in their internal tools. We show that, given a particular insurer's dataset, distortion risk measures other than that of the Value-at-Risk can provide the same risk estimates. However, if the insurer does chose a different risk measure, this provides complementary tools for evaluating risk that can be used to understand its position in the European insurance or financial market, or even to benchmark it in relation to the mandatory risk assessment standard.

The article is structured as follows. Section 2 is devoted to a brief presentation of distortion risk measures and indicators for Choquet integrals. Section 3 examines and discusses the tools to analyze implicit risk attitudes in distortion risk measures. Section 4 describes an application and its results, and 
outlines the strategy and the methodology used to calibrate risk measure parameters. The programming of the data analysis was carried out using the open source $\mathrm{R}$ statistical programming language and software. Finally, section 5 concludes.

\section{Distortion risk measures and aggregation indicators}

\subsection{Choquet integral and distortion risk measures}

We define the Choquet integral in line with Denneberg (1994). The (asymmetric) Choquet integral with respect to a set function $\mu$ of a $\mu$-measurable function $X: \Omega \rightarrow \overline{\mathbb{R}}$ is denoted as $\mathcal{C}_{\mu}(X)$ and is equal to

$$
\mathcal{C}_{\mu}(X)=\int X d \mu=\int_{-\infty}^{0}\left[S_{\mu, X}(x)-\mu(\Omega)\right] d x+\int_{0}^{+\infty} S_{\mu, X}(x) d x,
$$

if $\mu(\Omega)<\infty$, where $S_{\mu, X}(x)=\mu(\{X>x\})$ denotes the survival function of $X$ with respect to $\mu$. Note that $\Omega$ denotes a set, which in many applications is the sample space of a probability space. A set function $\mu$ in this context is a function defined from $2^{\Omega}$ (the set of all subsets of $\Omega$ ) to $\overline{\mathbb{R}}$. A $\mu$ measurable function $X$ is, widely speaking, a function defined on $\Omega$ so that expressions like $\mu(\{X>x\})$ or $\mu(\{X \leq x\})$ make sense. If $\mu$ is defined so that $0 \leq \mu(\Omega)<\infty$ and it also satisfies that $\mu(\emptyset)=0$ and that if $A \subseteq B$ then $\mu(A) \leq \mu(B)$, for any $A, B \in 2^{N}$ (monotonicity), then $\mu$ is often called a capacity.

The Choquet integral may be used in the definition of distortion risk measures as introduced by Wang (1995, 1996). A distortion function is a non-decreasing and injective function $g$ from $[0,1]$ to $[0,1]$ such that $g(0)=0$ and $g(1)=1$. Consider a probability space and the set of all random variables defined on this space. Given one of these random variables $X$, the value $\rho_{g}(X)$ that a distortion risk measure returns when applied to $X$ may be understood as the value of the asymmetric Choquet integral of $X$ with respect to a capacity, which is built by distorting the survival probability of $X$ with the distortion function $g$, i.e. $\rho_{g}(X)=\int X d(g \circ P)$.

Therefore, the distortion risk measure can be defined as $\rho_{g}(X)=\mathcal{C}_{\mu}(X)$ with $\mu=g \circ P$, as shown in (1). Note that the distortion function $g$ distorts the survival probability of $X$. The mathematical 
expectation of $X$ can be understood as a particular case of a distortion risk measure such that $\mathbb{E}(X)=$ $\mathcal{C}_{i d \circ P}(X)$, where the distortion function is the identity function $i d$. Indeed, the value of a distortion risk measure $\rho_{g}(X)$ may be interpreted as the expectation of $X$ given that the survival probability of $X$ has been previously distorted by the function $g$.

The most frequently used distortion risk measures are the quantile-based risk measures Value-at-Risk (VaR) and Tail Value-at-Risk (TVaR). Let us consider the $\mathrm{VaR}_{\alpha}$ and $\mathrm{TVaR}_{\alpha}$ risk measures that are defined for a random variable $X$ as $\operatorname{VaR}_{\alpha}(X)=\inf \left\{x \mid F_{X}(x) \geq \alpha\right\}=F_{X}^{-1}(\alpha)$ and $\operatorname{TVaR}_{\alpha}(X)=$ $\frac{1}{1-\alpha} \int_{\alpha}^{1} \operatorname{VaR}_{\lambda}(X) d \lambda$, respectively, where $0 \leq \alpha \leq 1$ is a confidence level. The relationship between the VaR and TVaR risk measures and discrete Choquet integrals has been described in literature (BellesSampera et al. 2013). Both $\operatorname{VaR}_{\alpha}(X)$ and $\operatorname{TVaR}_{\alpha}(X)$ may be understood as Choquet integrals with respect to capacities $\nu=\psi_{\alpha} \circ P$ and $\tau=\gamma_{\alpha} \circ P$, respectively, where $P$ is the probability function of $X$, $\psi_{\alpha}(u)=\mathbb{1}_{[1-\alpha, 1]}(u)$ and $\gamma_{\alpha}(u)=\frac{u}{1-\alpha} \cdot \mathbb{1}_{[0,1-\alpha)}(u)+\mathbb{1}_{[1-\alpha, 1]}(u)$ are the distortion functions associated with these risk measures, where $\mathbb{1}_{I}(u)$ denotes an indicator function which equals 1 when $u$ is in the interval $I$ and 0 otherwise.

Many articles have recently examined risk measures based on either distortion functions (Zhu and Li, 2012, Belles-Sampera et al., 2014a, Tsanakas and Millossovich, 2016) or generalizations of the quantiles (Bellini and Gianin, 2012, Bellini et al., 2014). The interplay between both of these two groups of risk measures has been examined (Dhaene et al., 2012, Goovaerts et al., 2012).

\subsection{GlueVaR distortion risk measures}

GlueVaR risk measures were introduced by Belles-Sampera et al. (2014a). These measures are defined by means of four parameters $\alpha, \beta, \omega_{1}$ and $\omega_{2}$, such that $0<\alpha \leq \beta<1, \frac{\beta-1}{\beta-\alpha} \leq \omega_{1} \leq 1$ and $\omega_{1}+\omega_{2} \leq 1$. It is shown by authors that the $\operatorname{GlueVaR}_{\beta, \alpha}^{\omega_{1}, \omega_{2}}$ is a distortion risk measure. In other words, GlueVaR $\mathrm{R}_{\beta, \alpha}^{\omega_{1}, \omega_{2}}(X)=\int X d \mu$ is the asymmetric Choquet integral of $X$ with respect to capacity $\mu=\kappa_{\beta, \alpha}^{\omega_{1}, \omega_{2}} \circ P$, where $\kappa_{\beta, \alpha}^{\omega_{1}, \omega_{2}}$ is the distortion function of a GlueVaR risk measure as shown in expression 
(2) and $P$ is the probability of the random variable $X$;

$$
\kappa_{\beta, \alpha}^{\omega_{1}, \omega_{2}}(u)=\left\{\begin{array}{lll}
{\left[\frac{\omega_{1}}{1-\beta}+\frac{\omega_{2}}{1-\alpha}\right] \cdot u} & \text { if } & 0 \leq u<1-\beta \\
\omega_{1}+\frac{\omega_{2}}{1-\alpha} \cdot u & \text { if } & 1-\beta \leq u<1-\alpha \\
1 & \text { if } & 1-\alpha \leq u \leq 1 .
\end{array}\right.
$$

An appealing feature of the $\mathrm{GlueVaR}_{\beta, \alpha}^{\omega_{1}, \omega_{2}}$ risk measure is that it can be expressed as a linear combination of three risk measures, as follows:

$$
\operatorname{GlueVaR}_{\beta, \alpha}^{\omega_{1}, \omega_{2}}(X)=\omega_{1} \cdot \operatorname{TVaR}_{\beta}(X)+\omega_{2} \cdot \operatorname{TVaR}_{\alpha}(X)+\left(1-\omega_{1}-\omega_{2}\right) \cdot \operatorname{VaR}_{\alpha}(X),
$$

with confidence levels $\beta$ and $\alpha$. From expression (3) it is immediately observed that VaR and TVaR can be defined as particular GlueVaR risk measures, i.e. $\mathrm{GlueVaR}_{\beta, \alpha}^{0,0}=\mathrm{VaR}_{\alpha}, \mathrm{GlueVaR}_{\beta, \alpha}^{0,1}=\mathrm{TVaR}_{\alpha}$ and $\mathrm{GlueVaR}_{\beta, \alpha}^{1,0}=\mathrm{TVaR}_{\beta}$. GlueVaR risk measures can be connected to the Tail Distortion risk measures, as defined by Zhu and Li (2012). The relationship between these risk measures, and a result regarding asymptotic features of GlueVaR for continuous heavy-tailed distributions, has been recently shown (Belles-Sampera et al. 2014b).

In terms of decision-making and risk management, GlueVaR risk measures combine three possible risk measurement scenarios: a highly tolerant to undesirable events scenario using $\mathrm{TVaR}_{\beta}$ as the risk measure; a tolerant scenario using $\mathrm{TVaR}_{\alpha}$; and a less tolerant scenario using $\mathrm{VaR}_{\alpha}$. As each GlueVaR combines these three risk scenarios, it allows different specific risk attitudes to be reflected. GlueVaR risk measures can be implemented by real world practitioners, because flexibility of these measures makes them useful in a variety of contexts. However, each particular risk context could require a different set of parameter values; thus, proposing a general criterion for GlueVaR calibration in all risk contexts is not possible. In next sections we suggest criteria for calibrating GlueVaR parameters and we examine their association with an attitude towards risk. 
2.3 Degree of orness of the Choquet integral

The Choquet integral can be regarded as a aggregation function. Aggregation functions are mathematical functions used for combining information in many fields of human knowledge, as artificial intelligence, biology or economics (Torra and Narukawa, 2007). Aggregation indicators are used to characterize the aggregation function. One of the most frequently used indicators is the degree of orness, which seeks to summarize the importance of each $i$ th-order statistic, $i=1, \ldots, n$, in the aggregation process associated with the Choquet integral with respect to capacity $\mu$. This indicator provides some kind of level of preference inherent to such an aggregation function on a $[0,1]$ scale, where 0 represents the minimum and 1 the maximum order statistic (Dujmović, 2006, Fernández Salido and Murakami, 2003).

Let $\mu$ be a capacity on $\Omega=\left\{\varpi_{1}, \ldots, \varpi_{n}\right\}$, and $X: \Omega \rightarrow \overline{\mathbb{R}}^{+}$be a function. If we assume that input data $\left(x_{1}, x_{2}, \ldots, x_{n}\right)$ are increasingly ordered, the expression (1) for discrete positive functions is equal to

$$
\mathcal{C}_{\mu}(f):=\sum_{i=1}^{n} x_{i}\left(\mu\left(A_{i}\right)-\mu\left(A_{i+1}\right)\right)
$$

Note that $A_{i}=\left\{\varpi_{i}, \cdots, \varpi_{n}\right\}$. Extensions of the Choquet integral of real functions (and not just positivereal functions) can be found in the literature (Mesiar et al. 2011). The degree of orness of the Choquet integral with respect to $\mu$ is computed as follows,

$$
\boldsymbol{\omega}\left(\mathcal{C}_{\mu}\right)=\sum_{i=1}^{n}\left(\frac{i-1}{n-1}\right) \cdot\left[\mathbb{S}_{n-i+1}^{\mu}-\mathbb{S}_{n-i}^{\mu}\right]
$$

where $\mathbb{S}_{i}^{\mu}=\mu\left(A_{n-i+1}\right)$. More details can be found in Belles-Sampera et al. (2014c). To simplify the notation, the difference $\mathbb{S}_{n-i+1}^{\mu}-\mathbb{S}_{n-i}^{\mu}$ is denoted as $w_{i}^{\mu}$, which can be understood as the weight indicating the importance given to the $i$-th order statistic represented by the fraction $\frac{i-1}{n-1}, i=1 \ldots n$. The degree of orness is one when the weight $w_{n}^{\mu}$ associated with the maximum is equal to one and the remaining weights are zero. It takes zero when the weight of the minimum $w_{1}^{\mu}$ is equal to one and the rest are zero. Thus, the value of the degree of orness indicates how the aggregation performed by the Choquet integral 
is ranked between these two boundaries.

It should be emphasized that the data ordering hypothesis can be relaxed. Indeed, the degree of orness can be computed without assuming the ordering of input data. Unfortunately, in practice, this flexible approach has high computational costs if $n$ is large. Finally, alternative aggregation indicators have been developed in the literature to characterize the Choquet integral. Examples include the divergence and the variance indicators (Belles-Sampera et al., 2014c).

\section{Risk attitude of agents}

We analyze here agents' attitudes to risk when they opt for a particular distortion risk measure to assess a risk. The degree of orness and a graphical analysis of the relative distortion associated with the risk measure are two useful tools for characterizing agents' risk attitudes. The first of these tools is a global indicator of their attitude towards losses. Their perception of risk is frequently not constant over the whole range of losses. An agent's risk attitude can therefore be examined in any interval of losses using a graphical inspection of the relative distortion associated with the risk measure.

3.1 Degree of orness and global risk attitude

The orness of the distorted probability and other quantifiers has been studied in the literature (Yager. 1999, Torra and Narukawa, 2007). A distortion risk measure may be understood as an aggregation operator because its relationship with the Choquet integral and, from the perspective of aggregation operators, the degree of orness is interpreted as an index that computes the similarity of the Choquet integral with the maximum (Yager, 1999, Torra and Narukawa, 2007). Adapting this interpretation to the risk measurement context, the degree of orness provides a level of similarity between the distorted survival function associated with the risk measure and the theoretical distorted survival function which returns the maximum loss. Note that the maximum value is returned in the worst-case scenario, i.e. the distorted survival function is equal to one in the range $(0,1]$ and zero in zero. The lower the degree of orness falls, 
the more dissimilar is the distorted survival function to the worst-case survival function. Therefore, an agent would be less risk tolerant in aggregated terms when the degree of orness is low.

An additional interpretation can be provided from the perspective of risk management. To provide some insights into the information on risk attitudes provided by the degree of orness, let us rewrite expression (5) as follows:

$$
\boldsymbol{\omega}\left(\mathcal{C}_{\mu}\right)=\sum_{i=1}^{n-1} \frac{1}{n-1} \cdot \mathbb{S}_{n-i}^{\mu}
$$

If $\mu=g \circ P$, it is straightforward to check that following expression holds,

$$
\mathbb{S}_{n-i}^{\mu}=g\left(S_{X}\left(x_{i}\right)\right)=g\left(\sum_{j=i+1}^{n} p_{j}\right)
$$

where $p_{j}$ is the probability of $x_{j}, j=1 \ldots n$. When combining expressions (6) and (7) it can be deduced that $\boldsymbol{\omega}\left(\mathcal{C}_{g \circ P}\right)$ provides an approximation to the area under the function $g$. The level of accuracy of the approximation increases as $n$ increases, where $n$ is the number of different values. Figure 1 illustrates how the degree of orness approximates the area under the function $g$ for the mathematical expectation (on the left) and the $\operatorname{VaR}_{\alpha}$ (on the right), where their distortion functions are plotted as dotted lines. The accuracy of the approximation depends on the size of $n$. The area under the function $g$ is computed by means of $n$ rectangles, where each rectangle has a width equal to $\frac{1}{n-1}$. So, a more accurate approximation of the area under $g$ would be given as $n$ increases, conditioned by the fact that a larger set of distinct values of $X$ is involved when $n$ increases.

As indicated previously, the mathematical expectation can be understood as a distortion risk measure involving the identity function $i d$ as the associated distortion function. The area under the $i d$ function is one half. Figure1 (left) illustrates that the degree of orness associated with the mathematical expectation should be approximately one half. In the case of the $\mathrm{VaR}_{\alpha}$ risk measure (Figure 1, right), the degree of orness linked to $\operatorname{VaR}_{\alpha}$ must be close to $\alpha$ for a large $n$, since $\alpha$ is the value of the area under the graph of $\psi_{\alpha}(u)=\mathbb{1}_{[1-\alpha, 1]}(u)$. 

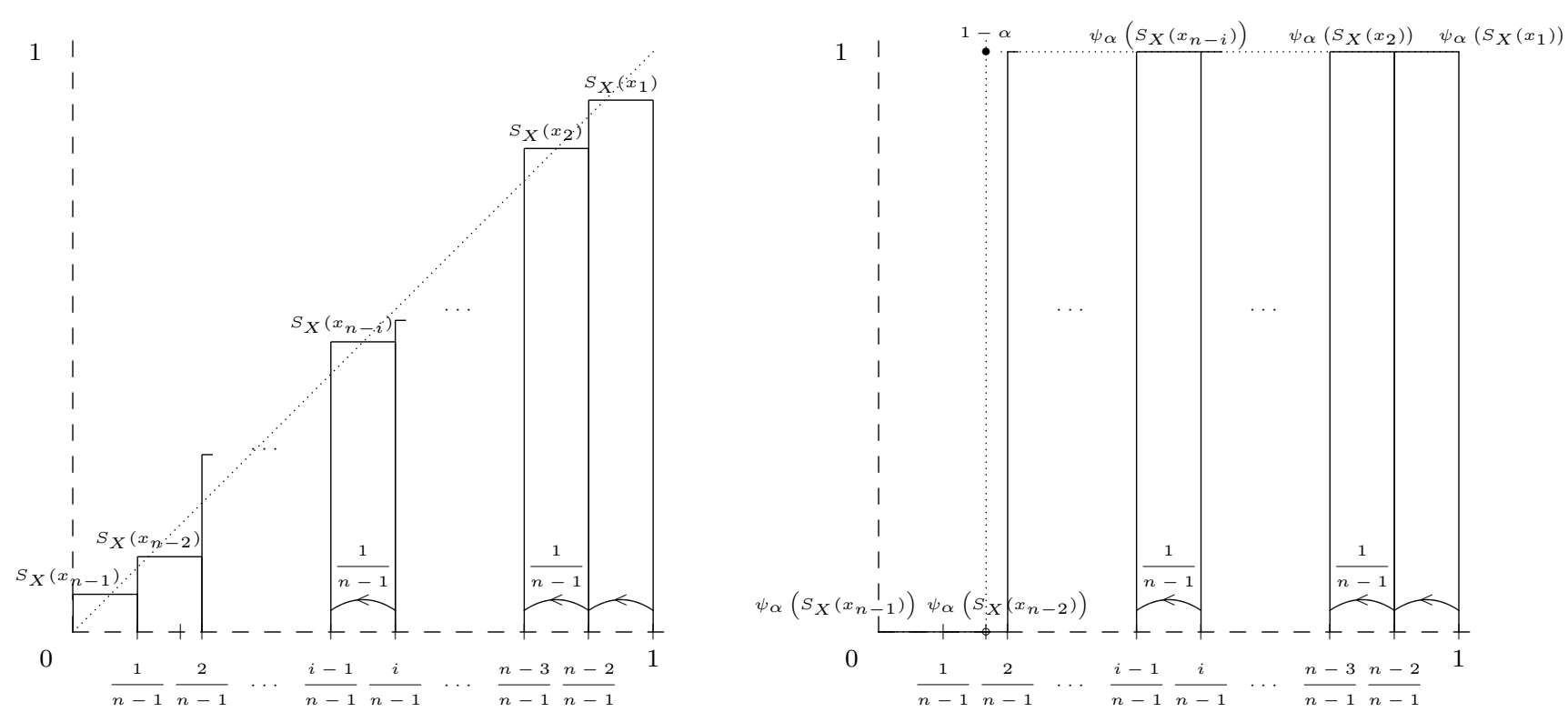

Fig. 1 Approximation to the area under the distortion function given by the degree of orness. Left: the identity function id in the case of the mathematical expectation. Right: distortion function of the $\operatorname{VaR}_{\alpha}$ risk measure, $\psi_{\alpha}(u)=\mathbb{1}_{[1-\alpha, 1]}(u)$. Note: $x_{1}, x_{2}, \ldots, x_{n}$ are increasingly ordered.

The approximations to the areas under the distortion functions of the $\mathrm{TVaR}_{\alpha}$ and the $\mathrm{GlueVaR}_{\beta, \alpha}^{\omega_{1}, \omega_{2}}$ risk measures given by their degrees of orness are here not plotted. In the case of the $\mathrm{TVaR}_{\alpha}$, however, it is easily deduced that its degree of orness should be approximately $\frac{1+\alpha}{2}$ which is the value of the area under $\gamma_{\alpha}(u)=\frac{u}{1-\alpha} \cdot \mathbb{1}_{[0,1-\alpha)}(u)+\mathbb{1}_{[1-\alpha, 1]}(u)$. Similarly, the area under the distortion function associated with the GlueVaR $\mathrm{R}_{\beta, \alpha}^{\omega_{1}, \omega_{2}}$ can be understood as a lineal combination of three different areas from expression (3), so its degree of orness should be close to $\alpha+\omega_{1} \cdot\left(\frac{1+\beta}{2}-\alpha\right)+\omega_{2} \cdot\left(\frac{1-\alpha}{2}\right)$.

Since the degree of orness provides an accurate approximation of the area under $g$, this quantity can be understood as an indicator of the global risk attitude of an agent, with decision-makers being classified as risk tolerant, risk neutral or risk intolerant. Note that we assume that a risk neutral agent would not distort the survival distribution function, so the associated distortion function linked to aggregated risk neutrality would be the $i d$ function. The area under the $i d$ function is one half and this value could be used as a benchmark of global risk attitude. A globally risk tolerant agent would make an upper distortion of the survival distribution in accumulated terms; thus, the area under $g$ for this agent would be larger 
than one half. Similarly, an agent would be globally risk intolerant if the area under $g$ was lower than one half. An agent would be globally risk tolerant using either the $\mathrm{VaR}_{\alpha}$ measure with $\alpha>0.5$ or the $\mathrm{TVaR}_{\alpha}$ with a positive $\alpha$. In the case of the $\mathrm{GlueVaR}_{\beta, \alpha}^{\omega_{1}, \omega_{2}}$, the implicit global risk attitude would depend on the values of the four parameters that define the risk measure.

3.2 Graphical analysis of local risk attitude

A graphical analysis is proposed to complement the examination of decision makers' risk attitudes when using distortion risk measures. While the degree of orness evaluates the accumulated distortion performed over the survival distribution function, it does not take into account which part of the survival distribution function was distorted. Clearly, from the perspective of a manager, distorting the survival probability in the right tail of the random variable linked to losses is not the same as distorting this probability in the left tail. Additionally, all distortion functions with an area equal to one half would be associated with global risk neutrality, where the $i d$ function is only a particular case.

One option open to us is to define the risk attitude in absolute terms. An absolute risk neutral agent is a decision-maker that does not distort the survival probability and who, therefore, uses the $i d$ function as the associated distortion function, i.e. $g(u)=i d(u)=u$ for all $0 \leq u \leq 1$. An absolute risk tolerant agent is associated with a distortion function $g$ such that $g(u)>u$, for all $0 \leq u \leq 1$. And, similarly, an absolute risk intolerant agent has a distortion function $g$ such that $g(u)<u$, for all $0 \leq u \leq 1$. This definition of risk attitude is in absolute terms in the sense that the relationship of ordering between $g(u)$ and $u$ must be fulfilled in the whole range $[0,1]$. Note that these considerations lead to a more restrictive definition of risk attitude than that provided by the global risk attitude.

The definition of the absolute risk attitude implies that the implicit attitude of an agent is invariant over the range of values. Yet, there are no reasons as to why the agent should have a unique risk attitude across the whole range. An agent's attitude to risk is likely to differ in accordance with the interval of loss under consideration. The risk attitude implicit in frequently used risk measures is not invariant; this 
is the case, for instance, of the $\mathrm{VaR}_{\alpha}$. When using the $\mathrm{VaR}_{\alpha}$, a risk tolerant attitude is associated with the interval $[1-\alpha, 1)$, but a risk intolerant attitude is associated with the interval $(0,1-\alpha)$. Thus, an homogeneous risk attitude cannot be linked to the $\mathrm{VaR}_{\alpha}$ risk measure throughout the domain.

Let us define the function $Q_{g}(u)$ on $u \in(0,1]$ as the quotient between the distortion function $g$ and the identity function, $Q_{g}(u)=\frac{g(u)}{u}$ for all $0<u \leq 1$. The $Q_{g}(u)$ allows the analysis of the agent's perception of risk at any point in the survival probability distribution. It takes non-negative values. This quotient function provides a function of survival probabilities, $u$, which describes the distortion factor applied by $g$ at each $u$ level. The quotient $Q_{g}$ is a quantifier of the local risk tolerance of the agent at any point. The quotient value represents the relative risk attitude of the decision maker compared to that of an agent with a risk neutral attitude who is confident of the survival probability. An agent is risk neutral, risk tolerant or risk intolerant at point $u$ if $Q_{g}(u)$ is equal to, higher or lower than one, respectively. The graphical analysis consists of plotting $Q_{g}(u)$, for all $0<u \leq 1$. Two examples are shown in Figure 2, where the quotient functions associated with the $\mathrm{VaR}_{\alpha}$ and $\mathrm{TVaR}_{\alpha}$ are displayed, $Q_{\psi_{\alpha}}$ and $Q_{\gamma_{\alpha}}$ respectively. The quotient function of the $\mathrm{GlueVaR}_{\beta, \alpha}^{\omega_{1}, \omega_{2}}$ is not plotted because the particular shape for the $Q_{\kappa_{\beta, \alpha}^{\omega_{1}, \omega_{2}}}$ depends on the values of the four parameters that define the risk measure.

We should emphasize that the quotient $\frac{1}{u}$, for all $0<u \leq 1$, marks out the maximum risk resistance frontier. Since the quotient function computes the ratio between the distorted survival probability and the survival probability, so $\frac{1}{u}$ is the maximum value attainable by this quotient function. Note that the quotient function is equal to 1 in the whole range for a completely risk neutral agent in absolute terms (absolute risk neutral agent). Thus, an agent's risk intolerance attitude emerges at point $u$ when the quotient function is smaller than one.

If we focus our attention on the quotient function associated with the VaR, $Q_{\psi_{\alpha}}$, it can be seen that a radical risk attitude is implicit in the interval $[1-\alpha, 1)$, shifting to the opposite extreme in the interval $(0,1-\alpha)$. Indeed, a maximum risk tolerance is involved in $[1-\alpha, 1)$ and a maximum risk intolerance attitude is involved in $(0,1-\alpha)$. Some similarities are found when the quotient function associated with the TVaR is examined, $Q_{\gamma_{\alpha}}$. Two ranges involving a different risk attitude are also distinguished. 

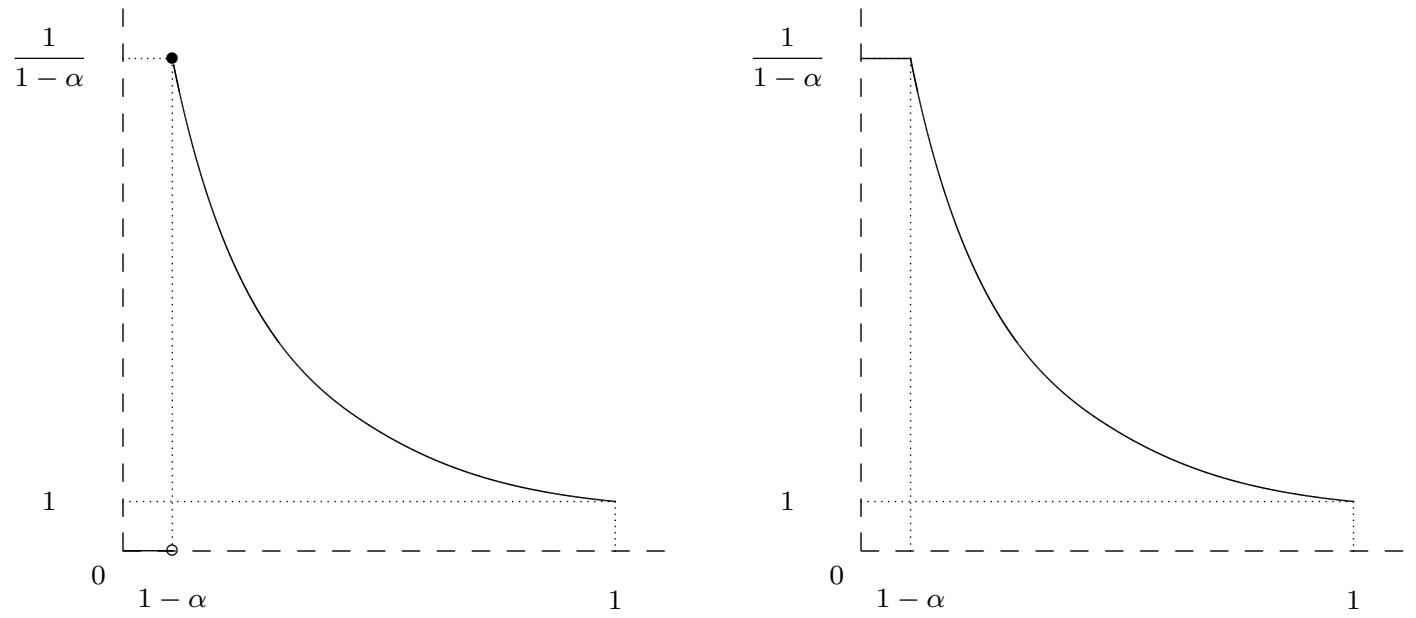

Fig. 2 The quotient function of $\operatorname{VaR}_{\alpha}, Q_{\psi_{\alpha}}$ (left) and the quotient function of TVaR ${ }_{\alpha}, Q_{\gamma_{\alpha}}$ (right). Note that they only differ in terms of the interval $[0,1-\alpha)$. The quotient function of the mathematical expectation, $Q_{i d}$, is represented by a horizontal dotted line.

Maximum risk tolerance is involved in the interval $[1-\alpha, 1)$ and a constant (non-boundary) risk tolerance attitude is involved in $(0,1-\alpha)$. Unlike the $\mathrm{VaR}_{\alpha}$, an absolute risk tolerance attitude is associated with the $\mathrm{TVaR}_{\alpha}$ because the quotient function is larger than one throughout the range $(0,1)$. As in the former two risk measures, a maximum risk resistance is involved in the interval $[1-\alpha, 1)$ when the quotient function of the GlueVaR $\mathrm{R}_{\beta, \alpha}^{\omega_{1}, \omega_{2}}$ is analyzed. However, more than just one attitude can be involved in the range $(0,1-\alpha)$. The high flexibility of $\mathrm{GlueVaR}_{\beta, \alpha}^{\omega_{1}, \omega_{2}}$ allows multiple attitudes towards risk to be implicit in the range $(0,1-\alpha)$, depending on the values of the remaining three parameters, $\beta, \omega_{1}$ and $\omega_{2}$.

In short, the quotient function $Q_{g}(u)$ can be used to characterize the relative risk behavior of an agent at any point. The value of a quotient function at a particular point depends on the distortion function as well as on on the original survival function. In other words, risk attitude in the quotient function is contemplated in the size of the distortion performed (the denominator) but also in the position in which this distortion is performed (the numerator). Note that the area under the quotient function provides similar information to that of the area under $g$, but expressed in terms of risk neutrality. Indeed, the area under the quotient function can be interpreted as the area under a weighted distortion function, where 
weights $(1 / u)$ are given to distorted values, i.e. $g(u) \cdot \frac{1}{u}$. Following this interpretation, greater weights are assigned to distortion function values associated with lower survival values.

The areas under the quotient function of $\mathrm{VaR}_{\alpha}$ and $\mathrm{TVaR}_{\alpha}$ are $A\left(Q_{\psi_{\alpha}}\right)=-\ln (1-\alpha)$ and $A\left(Q_{\gamma_{\alpha}}\right)=$ $1-\ln (1-\alpha)$, respectively. Similarly, the area under the quotient function of the $\mathrm{GlueVaR}_{\beta, \alpha}^{\omega_{1}, \omega_{2}}$ is equal to $A\left(Q_{\kappa_{\beta, \alpha} \omega_{1}, \omega_{2}}\right)=\omega_{1}\left[1+\ln \left(\frac{1-\alpha}{1-\beta}\right)\right]+\omega_{2}-\ln (1-\alpha)$.

Evaluating the area under the quotient function may be useful when analyzing the aggregate risk behavior in situations in which values of the distortion function are weighted, indicating that risk resistance is negatively associated with the size of the survival values. Thus, the area under the quotient function can be interpreted as a weighted quantifier of the aggregate risk attitude, where an area equal to one indicates global risk neutrality, an area larger than one indicates global risk resistance and an area lower than one indicates global risk intolerance.

\section{Application}

This section illustrates how the above findings can be applied in characterizing underlying risk attitudes - more specifically, it illustrates the challenges faced by European insurance companies. It is devised to highlight situations in which the implicit risk attitude linked to the VaR is unable to detect changes in potential catastrophic losses. We argue that the use of equivalent GlueVaR risk measures can be helpful in overcoming this drawback. The example involves the calibration of GlueVaR risk measures for a real dataset. Details regarding the calibration process to obtain equivalent GlueVaR risk measures are included in the Appendix.

4.1 Risk assessment in a scenario involving catastrophic losses

Suppose that the risk management function of a European insurance company is involved in its Own Risk and Solvency Assessment (ORSA), in accordance with Directive 2009/138/EC of 25 November 2009 (known as the Solvency II Directive), or in the design of a total or partial internal model for the company under the same regulatory framework. In this framework, the VaR with a confidence level $\alpha=99.5 \%$ 
is required to assess the regulatory capital. Note that the selection of the confidence level involves a trade-off between protection and competitiveness. The level of the protection could be reduced with low confidence levels. An increase in the confidence levels could involve higher economic reserves and, therefore, the protection would rise; however, this could also affect the competitiveness.

Risk managers may dislike using the VaR as a risk measure, because of the lack of risk-based information it provides on catastrophic losses. Indeed, two firms with marked differences in the sum of their potential losses in adverse scenarios may report the same risk value, even though they are not exposed to the same level of risk. As such, their disparities would go unobserved by decision makers. Moreover, the lack of subadditivity may well constitute another drawback. Alternatives to $\mathrm{VaR}_{99.5 \%}$ that take into account catastrophic losses can be considered by risk managers. Traditional approaches frequently lead to severely higher economic reserves. Managers need to find a risk measure that generates similar economic reserves than $\mathrm{VaR}_{99.5 \%}$ for the overall risk faced by the insurance company and, additionally, they would like that the alternative risk measure provides risk-based information on catastrophic losses and that, hopefully, it satisfies appealing subadditivity properties. This is a real practical problem because the Solvency II Directive allows insurance undertakings to look for such alternatives 1 .

\subsection{Data and Results}

In the example two loss random variables $X_{1}$ and $X_{2}$ affect a motor insurance company. Motor claim cost data provided by a leading Spanish motor insurer are used to illustrate the calibration procedure. The sample consists of $n=518$ observations of individual claim costs (in thousands of euros). These data were analyzed in previous studies (Bolancé et al., 2008, Guillén et al., 2011). The dataset contains $X_{1}$ and $X_{2}$, which report property damage costs and bodily injury costs, respectively. Total claim costs are the sum of the two, $Z=X_{1}+X_{2}$. The aggregate risk faced by the insurer is the sum of both random variables. We assume that the insurer uses the $\mathrm{VaR}_{99.5 \%}$ as its risk measure.

\footnotetext{
1 See Article 122(1) of the Directive.
} 
Before dealing with the calibration of the GlueVaR measures, we first compute the $\operatorname{VaR}_{99.5 \%}(Z)$ and its associated degree of orness. The risk measure value is equal to $\operatorname{VaR}_{99.5 \%}(Z)=187.612$ and the degree of orness is equal to $\boldsymbol{\omega}\left(\mathrm{VaR}_{99.5 \%}\right) \simeq 0.996$. Let us now modify the original dataset so that catastrophic losses are artificially increased. Specifically, the two pairs of bivariate losses $\left(x_{1}, x_{2}\right)$ that lead to the highest aggregated loss $z$ are each multiplied by 10. Total claim costs in the modified dataset are represented by the random variable $Z^{*}$, and the value of the $\operatorname{VaR}_{99.5 \%}\left(Z^{*}\right)$ and the associated degree of orness are computed. We find that values of $\mathrm{VaR}_{99.5 \%}$ and $\boldsymbol{\omega}$ are equal in the two scenarios, both for the original and the modified datasets (note that probabilities and $n$ remain unchanged and only $Z$ values located in the local risk intolerance zone of $\mathrm{VaR}_{99.5 \%}$ have been modified). Based on this information, a risk manager comfortable with the implicit risk attitude of $\mathrm{VaR}_{99.5 \%}$ is unaware that he faces a notable increase in catastrophic risk (or, to some extent, it might be concluded that a less risk resistant agent is involved in the second scenario).

Let us now focus on the strategy used to calibrate the GlueVaR parameters. The following steps are performed to obtain GlueVaR risk measures that are comparable to the $\operatorname{VaR}_{99.5 \%}(Z)$ : a) the minimum and maximum values of confidence levels are fixed at $90 \%$ and $99.9 \%$, i.e. $\alpha_{\min }=90 \%$ and $\beta_{\max }=99.9 \%$; b) the number of partitions is stipulated in $d=25$, so we deal with 625 optimization problems; c) the empirical distribution function of total claim costs is used for the risk quantification, and, finally, d) the outcome choice of the GlueVaR solutions are obtained using constrOptim function from rootSolve library in $\mathrm{R}$. The same phases are repeated for the modified random variable $Z^{*}$.

We found a set of optimal GlueVaR risk measures that give the same risk value as the $\mathrm{VaR}_{99.5 \%}$ in these two specific contexts. Thus, 341 optimal solutions were found for the random variable $Z$ and 605 for $Z^{*}$. Once a set of GlueVaR measures has been obtained, the degree of orness was computed to characterize the underlying aggregated risk attitude. The boundary values of the degree of orness and the associated GlueVaR measures were identified. We should emphasize that the maximum degree of orness was equal to the degree of orness of the $\mathrm{VaR}_{99.5 \%}$ in both scenarios. In fact, the optimal GlueVaR $\mathrm{G}_{\beta, \alpha}^{\omega_{1}, \omega_{2}}$ solution with the highest degree of orness was the GlueVaR with parameters $\alpha=99.5 \%, \beta=99.9 \%$ and 
$\omega_{1}=\omega_{2}=0$, and it holds that GlueVaR ${ }_{99.9 \%, 99.5 \%}^{0,0}=\mathrm{VaR}_{99.5 \%}$ (see expression (3)). In other words, given a certain risk value, the $\mathrm{VaR}_{\alpha}$ is the GlueVaR risk measure that presents the highest area under the associated distortion function of all the GlueVaR risk measures that return this value 2 .

The minimum degree of orness and the associated GlueVaR measure for the original dataset and the dataset with catastrophes are reported in Table 1. Information about the underlying aggregate risk attitude of the agent can be inferred from the minimum degree of orness. Table 1 shows that, for the original dataset, there exists an optimal GlueVaR risk measure for which the area of the associated distortion function is approximately 0.912. Thus, this GlueVaR risk measure gives the same value as that given by $\operatorname{VaR}_{99.5 \%}$ when applied to $Z$, but, in aggregate terms, it involves a more moderate distortion of the original survival distribution function, a lower degree of orness and, consequently, less global risk tolerance. Moreover, when assessing the data with catastrophic extremes, the minimum degree of orness is 0.896 indicating that the underlying global risk resistance of the agent could be even lower whether the $\mathrm{VaR}_{99.5 \%}$ risk measure is applied to $Z^{*}$. This finding is consistent with prior expectations because the modified-data scenario is a more risky scenario involving larger catastrophic losses. A remark is worth to make here. In the original-data scenario an optimal GlueVaR solution was found equivalent to the TVaR, but its associated degree of orness was not a boundary value 3 In the modified-data scenario, the TVaR was not found to be an optimal GlueVaR solution.

The degree of orness should be complemented with the examination of the quotient function which allows the relative risk attitude at any point of the survival distribution to be analyzed. The quotient functions of risk measures associated with boundary degrees of orness in both scenarios are examined. All the quotient functions analyzed are located in the upper risk-tolerance frontier in the range $[0.10,1)$. For ease of comparison, the quotient functions are rescaled and their left-tails are shown only in the range $[0,0.10]$ in Figure 3 .

\footnotetext{
2 Note that the distortion function associated with the $\operatorname{VaR}_{\alpha}$ assigns one to survival values higher than $(1-\alpha)$ and zero to the rest.

3 The GlueVaR ${ }_{97.4 \%, 90 \%}^{1,0}$ was an optimal solution with degree of orness equal to 0.986, and, as expressed in 3 , this measure is equivalent to the $\operatorname{TVaR}_{97.4 \%}$.
} 
Original dataset Dataset with

\begin{tabular}{lcc} 
& & Dataset with \\
& & catastrophic observations \\
\hline$\alpha$ & 0.900 & 0.900 \\
$\beta$ & 0.999 & 0.995 \\
$\omega_{1}$ & 0.188 & 0.039 \\
$\omega_{2}$ & $1.210^{-6} \cdot 10^{-6}$ & $0.812 \cdot 10^{-6}$ \\
\hline Degree of orness & 0.912 & 0.896 \\
\hline
\end{tabular}

Table 1 Parameter values of the associated GlueVaR measure equivalent to $\mathrm{VaR}_{99.5 \%}$ and minimum degree of orness for $Z$ (original dataset) and $Z^{*}$ (modified dataset).

Notable differences can be observed in the relative risk attitudes locally implicit in the left-tail of the quotient functions. Let us first examine the quotient functions of the GlueVaR risk measures that present the maximum degree of orness (left), which is the same quotient function associated with the $\mathrm{VaR}_{99.5 \%}$. The agent is most risk tolerant at any point of the interval $[0.5 \%, 1)$ and maximum risk intolerant at $(0,0.5 \%)$. This means, the quotient function is located in the upper frontier at $[0.5 \%, 1)$ and in the lower frontier at $(0,0.5 \%)$. When the GlueVaR measures that present the minimum degree of orness are analyzed for the equivalent GlueVaR (right), the patterns of the left-tails of the quotient functions are undoubtedly different. An interesting finding is that the $Q_{g}(u)$ is not located within the boundaries at any point of the interval $(0,0.10)$. This means, the risk tolerant attitude is not maximized in the range $[0.5 \%, 0.10)$ but, on the contrary, the agent is more risk resistant to catastrophic losses at $(0,0.5 \%)$ than when using $\operatorname{VaR}_{99.5 \%}$.

The differences in implicit risk attitudes between scenarios should be commented upon. In line with our initial beliefs, the agent's risk resistance is lower in the scenario that involves higher catastrophic losses. This can be interpreted as a warning that either a change in the agent's risk attitude or a change in the risk faced has been detected. The same conclusions are drawn when evaluating the area under the quotient function, where the areas take the values of 3.054 and 2.458 for the original scenario and the scenario with catastrophic losses, respectively. Note that the area under the quotient function of $\mathrm{VaR}_{99.5 \%}$ is equal to 5.298 . So, if the area under the quotient function is considered a weighted quantifier of the 

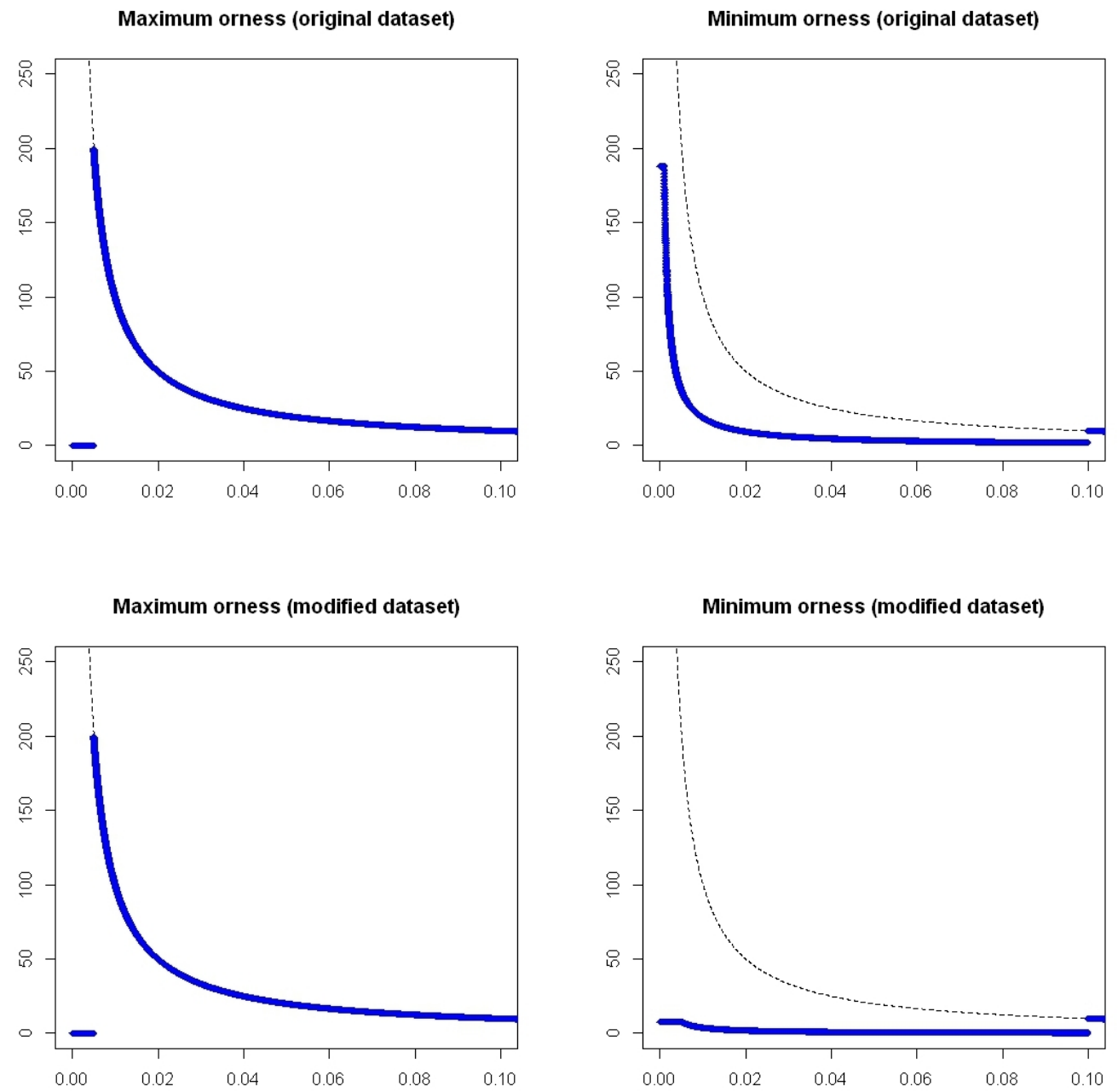

Fig. 3 Quotient functions of optimal solutions with maximum (left) and minimum (right) degrees of orness in the original dataset (upper) and the modified dataset with catastrophic observations (lower). Note: Dashed curve indicates the upperbound quotient function curve, i.e. $\frac{1}{u}$ for all $0 \leq u \leq 1$.

global risk attitude, the differences between the risk attitude involved in $\mathrm{VaR}_{99.5 \%}$ and the risk attitude implicit in the equivalent GlueVaR $\omega_{\beta, \alpha}^{\omega_{1}, \omega_{2}}$ measures become more evident in these two scenarios.

\section{Discussion}

Distortion risk measures are widely used for assessing risk in a range of different contexts. The risk value obtained from such measures depends basically on the characteristics of the random variable, which are 
captured by the survival distribution, and the associated distortion function. These two elements can be disaggregated and, here, our attention has focused on an analysis of the distortion function, which can be understood as a weighting function of survival probabilities. Thus, any risk attitude implicit in a distortion risk measure is to some extent attached to its distortion function. An aggregation indicator and a quotient function are used to describe alternative features of the distortion function. Here, we show that these mathematical devices provide valuable insights into the implicit risk tolerance associated with the choice of a risk measure.

Distortion risk measures can be understood as belonging to a class of aggregation functions and a set of indicators has been developed in the literature to characterize this class. In this article, we have analyzed the degree of orness and we showed that this aggregation indicator may be interpreted as an approximation of the area under the distortion function of the risk measure. The size of this area reveals the aggregate distortion made over the survival distribution. This being the case, this indicator reveals the global risk attitude when a particular distortion risk measure is applied. The analysis reported here allows to conclude that the degree of orness and the area under the distortion function can be interchangeably used to study an agent's global vision of risk.

The risk attitude implicit in a risk measure, however, is not only determined by the area of the distortion function - of equal importance is the distribution of this total distortion across the range of values. The risk information provided by the aggregation indicators needs to be complemented by the quotient function, defined as the ratio between the distortion and the identity functions. We demonstrate that a graphical analysis of this quotient function provides local information about the relative risk behavior associated with the risk measure at any point in the range of values. Additionally, the area under the quotient function provides similar aggregate risk information to that of the area under the distortion function, which means the former could replace the distortion function in analyses of global risk attitude, especially if distorted values need to be weighted.

The analysis of the risk attitude implicit in the Value-at-Risk measure illustrates that it is not sensitive to changes involving more risky scenarios. The reason for this is that the risk measure presents two extreme 
risk attitudes, i.e. maximum risk resistance in $[1-\alpha, 1)$ and maximum risk intolerance in $(0,1-\alpha)$. Here, we have shown that additional risk information may be obtained using comparable GlueVaR risk measures that are calibrated to report the same risk value as that of the $\mathrm{VaR}_{\alpha}$. The distortion functions of these GlueVaR risk measures have been examined and we showed that the calculation of the aggregation indicators and quotient functions of these GlueVaR measures gives a more complex characterization of the risk framework providing, for instance, boundary risk attitudes. Thus, we believe GlueVaR measures can usefully be employed by risk managers to enrich the risk information provided by the standard quantile, gaining a deeper understanding of the risks taken.

The calibration procedure of GlueVaR parameters was not the primary focus of this study and it was not our intention to cover all the possible calibration criteria. However, two final remarks are worth recording. First, the procedure proposed depends necessarily on the risk measure of reference and also on the random variable. As such, the set of comparable GlueVaR measures differs when the random variable changes. This is not an unbridgeable drawback when requesting that the supervisory authorities change the risk measure if decision makers are able to justify the GlueVaR selection process. For example, these measures may be used to analyze variations from one year to next in the implicit risk attitude of boundary cases when the $\mathrm{VaR}_{\alpha}$ is applied to assess the annual risk. Second, the $\mathrm{VaR}_{\alpha}$ was chosen as the risk measure of reference because of its application in practice, but the calibration strategy of the GlueVaR measures could easily be adapted to other risk measurement problems. Similarly, indicators other than the degree of orness might be considered in the GlueVaR measure choice. For instance, optimal GlueVaR solutions with the associated minimum and maximum areas under the quotient functions could be selected to reflect extreme risk attitudes of decision-makers.

All risk attitude concepts explored in the article are based on the characteristics of the distortion function employed by the risk measure that evaluates the random variable. However, most risk aversion definitions found in the literature are based on the characteristics of the utility function associated to the decision-maker, such as the von Neumann-Morgenstern or local-type utility functions (expected utility theory). The fundamental difference between both approaches is that the distortion function is applied 
over the distribution function of the random variable, whilst the utility function is directly applied over the random variable. So, our risk characterization would be more close to the Yaari's dual theory. To conclude, the risk attitude indicators could be used in the finance theory. Distortion functions have been recently applied in the context of two-price economic equilibrium (Madan and Cherny, 2010, Madan and Schoutens, 2012). The analysis of the implicit risk attitude could provide insights in the size of the cone in the bid-ask pricing.

Acknowledgements The authors acknowledge the valuable comments and suggestions from the referees and the managing editor. We acknowledge support received from the Spanish Ministry for grants ECO2013-48326-C2-1-P and ECO2015-66314R. MG thanks ICREA Academia. 


\section{References}

C. Alexander and J. M. Sarabia. Quantile uncertainty and value-at-risk model risk. Risk Analysis, 32 (8):1293-1308, 2012. ISSN 1539-6924. doi: 10.1111/j.1539-6924.2012.01824.x.

G. Beliakov, A. Pradera, and T. Calvo. Aggregation Functions: A Guide to Practitioners. Springer, Berlin, 2007. ISBN 978-3-540-73720-9.

J. Belles-Sampera, J. M. Merigó, M. Guillén, and M. Santolino. The connection between distortion risk measures and ordered weighted averaging operators. Insurance: Mathematics and Economics, 52(2): 411-420, 2013.

J. Belles-Sampera, M. Guillén, and M. Santolino. Beyond Value-at-Risk: GlueVaR distortion risk measures. Risk Analysis, 34(1):121-134, 2014a. doi: $\{10.1111 /$ risa.12080\}.

J. Belles-Sampera, M. Guillén, and M. Santolino. Relationship between GlueVaR and Tail distortion risk measures. Riskcenter Working Paper, 2014b.

J. Belles-Sampera, J. M. Merigó, M. Guillén, and M. Santolino. Indicators for the characterization of discrete Choquet integrals. Information Sciences, 267:201-216, 2014c. doi: $\{10.1111 /$ risa.12080\}.

F. Bellini and E. R. Gianin. Haezendonck-Goovaerts risk measures and Orlicz quantiles. Insurance: Mathematics and Economics, 51(1):107-114, JUL 2012. ISSN 0167-6687. doi: \{10.1016/j.insmatheco. $2012.03 .005\}$.

F. Bellini, B. Klar, A. Mueller, and E. R. Gianin. Generalized quantiles as risk measures. Insurance: Mathematics and Economics, 54:41-48, JAN 2014. ISSN 0167-6687. doi: \{10.1016/j.insmatheco.2013. $10.015\}$.

C. Bolancé, M. Guillén, E. Pelican, and R. Vernic. Skewed bivariate models and nonparametric estimation for the CTE risk measure. Insurance: Mathematics and Economics, 43(3):386-393, 2008. doi: 10.1016/ j.insmatheco.2009.05.010.

Cox Jr., L.A. Improving Risk Analysis. Springer-Verlag New York, 2013.

D. Denneberg. Non-Additive Measure and Integral. Kluwer Academic Publishers, Dordrecht, 1994. 
J. Dhaene, A. Kukush, D. Linders, and Q. Tang. Remarks on quantiles and distortion risk measures. European Actuarial Journal, 2(2):319-328, 2012.

J. J. Dujmović. A comparison of Andness/Orness Indicators. In Proceedings of the 11th Information Processing and Management of Uncertainty international (IPMU 2006), pages 691-698, 2006. ISBN 2-84254-112-X.

J. Fernández Salido and S. Murakami. Extending Yager's orness concept for the OWA aggregators to other mean operators. Fuzzy Sets and Systems, 139(3):515-542, 2003. ISSN 0165-0114.

M. Goovaerts, D. Linders, K. Van Weert, and F. Tank. On the interplay between distortion, mean value and Haezendonck-Goovaerts risk measures. Insurance: Mathematics and Economics, 51(1):10-18, 2012. ISSN 0167-6687. doi: 10.1016/j.insmatheco.2012.02.012.

M. Grabisch, J.-L. Marichal, R. Mesiar, and E. Pap. Aggregation functions, volume 127 of Encyclopedia of Mathematics and its Applications. Cambridge University Press, 2009.

M. Grigorova. Stochastic dominance with respect to a capacity and risk measures. Statistics and Risk Modeling, 31:259-295, 2014.

M. Guillén, F. Prieto, and J. M. Sarabia. Modelling losses and locating the tail with the Pareto Positive Stable distribution. Insurance: Mathematics and Economics, 49(3):454-461, 2011. doi: 10.106/j.insmatheco.2011.07.004.

I. Kojadinovic, J.-L. Marichal, and M. Roubens. An axiomatic approach to the definition of the entropy of a discrete Choquet capacity. Information Sciences, 172(1-2):131-153, 2005. ISSN 0020-0255. doi: 10.1016/j.ins.2004.05.011.

C. MacKenzie. Summarizing risk using risk measures and risk indices. Risk Analsys, 34(34):2143-62, 2014 .

D. Madan and A. Cherny. Markets as a counterparty: An introduction to conic finance. International Journal of Theoretical and Applied Finance, 13(08):1149-1177, 2010.

D. Madan and W. Schoutens. Structured products equilibria in conic two price markets. Mathematics and Financial Economics, 6(01):37-57, 2012. 
J.-L. Marichal. Tolerant or intolerant character of interacting criteria in aggregation by the Choquet integral. European Journal of Operational Research, 155(3):771-791, 2004. ISSN 0377-2217. doi: 10.1016/S0377-2217(02)00885-8.

R. Mesiar, A. Mesiarová-Zemánková, and K. Ahmad. Discrete Choquet integral and some of its symmetric extensions. Fuzzy Sets and Systems, 184(1):148-155, 2011. ISSN 0165-0114. doi: 10.1016/j.fss.2010.11. 013.

V. Torra and Y. Narukawa. Modeling Decisions: Information Fusion and Aggregation Operators. Springer, Berlin, 2007. ISBN 978-3-540-68789-4.

A. Tsanakas and P. Millossovich. Sensitivity Analysis Using Risk Measures. Risk Analysis, 36(1):30-48, 2016.

S. S. Wang. Insurance pricing and increased limits ratemaking by proportional hazard transforms. Insurance: Mathematics and Economics, 17(1):43-54, 1995. ISSN 0167-6687.

S. S. Wang. Premium calculation by transforming the layer premium density. ASTIN Bulletin, 26(1): $71-92,1996$.

R. R. Yager. Nonmonotonic owa operators. Soft Computing, 3(3):187-196, 1999. ISSN 1432-7643. doi: 10.1007/s005000050068. URL http://dx.doi.org/10.1007/s005000050068.

R. R. Yager. On the entropy of fuzzy measures. IEEE Transactions on Fuzzy Systems, 8(4):453-461, 2000. ISSN 1063-6706. doi: 10.1109/91.868951.

L. Zhu and H. Li. Tail distortion risk and its asymptotic analysis. Insurance: Mathematics and Economics, 51(1):115-121, JUL 2012. ISSN 0167-6687. doi: 10.1016/j.insmatheco.2012.03.010. 


\section{Appendix. Calibration of GlueVaR parameters}

To apply the GlueVaR $\mathrm{R}_{\beta, \alpha}^{\omega_{1}, \omega_{2}}$ risk measures values need to be assigned to the parameters defining the risk measure. All the steps required in calibrating GlueVaR risk measures are described here. The criterion followed in the calibration procedure is the need to obtain the same risk measure value with the GlueVaR measures as obtained with the $\mathrm{VaR}_{99.5 \%}$. Moreover, the selection of the risk measure is restricted to the subfamily of candidates to be tail-subadditive GlueVaR measures (Belles-Sampera et al., 2014a). The strategy for calibrating the parameters is as follows:

- Minimum and maximum admissible values of the $\alpha$ and $\beta$ confidence levels have to be determined, $\alpha_{\min }$ and $\beta_{\max }$

- Let us assume that $Z$ random variable represents the overall risk. A set of $d \times d$ constrained optimization problems is defined at this step:

$$
\begin{aligned}
\mathrm{P}_{i, j}: \min _{\omega_{1}, \omega_{2}} \mid \operatorname{GlueVaR} & \operatorname{SaR}_{\beta_{j}, \alpha_{i}}^{\omega_{1}, \omega_{2}}(Z)-\operatorname{VaR}_{99.5 \%}(Z) \mid, \\
& \text { subject to } \\
& \left\{\begin{array}{l}
0 \leq \omega_{1} \leq 1 \\
0 \leq \omega_{2} \\
\omega_{1}+\omega_{2} \leq 1
\end{array}\right.
\end{aligned}
$$

where $i, j=1, \ldots d, \alpha_{i}=\alpha_{\min }+\frac{i-1}{d-1}\left(\beta_{\max }-\alpha_{\min }\right), \beta_{j}=\alpha_{i}+\frac{j-1}{d-1}\left(\beta_{\max }-\alpha_{i}\right)$. Flexibility rises with the number of partitions $d$, as do computational costs. Constraints are fixed to guarantee that the GlueVaR can satisfy $\alpha_{i}$-tail subadditivity.

- An optimization algorithm should be used to solve this set of problems. If $\mathrm{P}_{i^{*}, j^{*}}$ represents the problem for which the minimum value of the objective function is reached and $\left(\omega_{1}^{*}, \omega_{2}^{*}\right)$ is the associated solution,

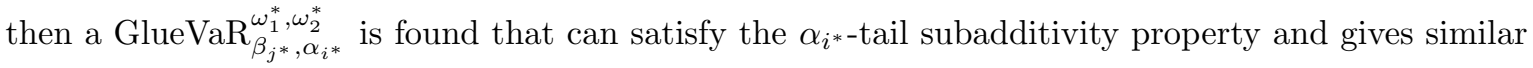
reserve values to those obtained with $\mathrm{VaR}_{99.5 \%}$ when applied to the overall risk of the company. $\mathrm{P}_{i, j}$ problems may not have solutions. Were this to be the case, then the optimization criteria would have to be revised, including a lower $\alpha_{\min }$, a higher $\beta_{\max }$ and/or a larger $d$. 
- More than one GlueVaR solution is frequently found. Alternative combinations of parameter values return the same objective function value, or a value that differs insignificantly. In this situation, solutions could be ranked in accordance with the underlying risk attitude involved. Aggregation indicators are useful for characterizing the aggregate underlying risk attitude of the agent when using each risk measure. Here, we propose ranking the solutions based on the value of the aggregation indicators associated with each optimal risk measure. With this goal in mind, degrees of orness are computed for (multiple) optimal GlueVaR $\operatorname{\beta aj}_{\beta^{*}, \alpha_{i^{*}}}^{\omega_{1}^{*}, \omega_{2}^{*}}$ solutions. Two particular GlueVaR measures among the set of solutions are of special interest:

Lower-limit solution. Selection of the GlueVaR risk measure with the associated minimum degree of orness;

Upper-limit solution. Selection of the GlueVaR risk measure with the associated maximum degree of orness.

In other words, boundaries of the degree of orness are detected. Optimal GlueVaR risk measures linked to boundaries reflect the extreme risk attitudes of agents when the random variable $Z$ is analyzed. 